\title{
Search of the Optimal Position of Sails
}

\section{Recherche de la Position Optimale des Voiles}

\author{
MADI Rafik ${ }^{1}$, GUENFOUD Mohamed ${ }^{1}$ \\ ${ }^{1}$ Université 8 Mai 1945, Génie Civil et Hydraulique, B. P. 401, Guelma, Algerie, madirafik2000@yahoo.fr
}

\begin{abstract}
During its history, Algeria has suffered several destructive earthquakes. According to the observations made during these earthquakes, the Algerian seismic regulation: RPA 82, RPA 83 and RPA 99 revised en 2003, seems insufficient and the buildings have suffered considerable damage. Which explains the presence of failures and insufficiencies in the Algerian seismic code.To resolve this problem, several researchers have advocated the introduction of bracing sails to improve the behavior of structures against the earthquake. In the Algerian seismic regulation, no article is related to this parameter. The objective sought in this study is to study the influence of the position of bracing sails on the behavior of structures across: the ductility demand $\mu_{D}$, inter-floor displacement $D_{p}$ and stiffness $K_{p}$ at the performance point, the elastic stiffness $K_{e}$ and the overall degradation indicator of the structure $I_{d}$. the results obtained showed that the best arrangement of the bracing sails is that which are placed at the end of the structure with perfect symmetry. Keywords : sails bracing, seismic design, Push-over analysis, modelization, optimization.
\end{abstract}

Résumé. Au cours de son histoire, l'Algérie a subi plusieurs séismes destructeurs. Selon les observations faites durant ces tremblements de terre, la règlementation parasismique algérienne : RPA 82, RPA 83 et RPA 99 révisé en 2003, parait insuffisante et les constructions ont subi des dommages considérables. Ce qui explique la présence de défaillances et insuffisances dans le code parasismique algérien. Pour résoudre ce problème, plusieurs chercheurs ont préconisé l'introduction des voiles de contreventement pour améliorer le comportement des structures vis-à-vis du séisme. Dans le règlement parasismique algérien, aucun article n'est relatif à ce paramètre. L'objectif recherché dans cette étude est d'étudier l'influence de la position des voiles de contreventements sur le comportement des structures à travers: la demande en ductilité $\mu_{D}$, le déplacement inter-étage $D_{p}$ et la raideur $K_{p}$ au point de performance, la raideur élastique $K_{e}$ et l'indicateur de dégradation globale de la structure $I_{d}$. les résultats obtenus ont montré que la meilleure disposition des voiles de contreventements est celle qui sont placées à l'extrémité de la structure avec une symétrie parfaite. Mots Clés : voiles de contreventement, conception parasismique, Analyse Push-over, modélisation, optimisation.

\section{Introduction}

Dans le context sismique, les meilleurs projets en termes de sécurité sont obtenus en respectant les principes de conception. La sécurité est en général mieux assurée dans une structure bien conçue est calculée de manière approchée que dans une structure mal conçue pour laquelle des calculs compliqués sont effectués. Les règlements parasismiques encouragent le concepteur à adopter des dispositions de conceptions en général favorables à un bon comportement de la structure. L'objectif à atteindre des projets de construction parasismiques, est de définir une structure capable de subir, sans s'effondrer, les déformations engendrées par l'action sismique. Cet objectif peut être atteint avec succès, par des projets de différents types d'ossatures et de divers degrés de capacité de dissipation d'énergie. Le respect des principes de construction laisse encore le concepteur du projet devant plusieurs choix à effectuer au préalable, car plusieurs solutions sont possibles qui respectent toutes les principes de conception parasismique. Suite au séisme qui a frappé la région du centre et en grande partie la wilaya de Boumerdes, des conclusions ont été tirées suite aux expertises qui ont été 
établies, que les constructions en voile en béton armé ont prouvées de leurs efficacités structurelles aux actions sismiques, dans les zones sinistrées $[1,2]$.

Les structures à voiles en béton armé présentent, particulièrement en zone sismique, plusieurs avantages: leur résistance limite les déformations latérales, leur rigidité permet de protéger les éléments non structuraux et quelques poteaux existants, leur présence permet de s'affranchir du difficile problème posé par la réalisation du ferraillage des nœuds des portiques, elles permettent de ne pas être pénalisées dans le choix du coefficient de comportement en cas de panneaux de remplissage.

Par rapport à d'autres systèmes constructifs tels que les portiques auto stable en poteaux- poutres, des dégâts et effondrements ont été observés pour de multiples raisons: mauvaise conception, sous dimensionnement des poteaux, manque d'armatures transversales dans les zones critiques des éléments structuraux, etc. Ce sont ces raisons qui ont conduit principalement, à pénaliser ce type de système structurel et principalement la limitation de la hauteur des structures. Les critères de vérification prisent en considération dans cette recherche sont les suivants: le déplacement inter-étages maximal $D_{\max }$, la demande en ductilité $\boldsymbol{\mu}_{\boldsymbol{D}}$, la raideur de la structure au point de performance $\boldsymbol{K}_{\boldsymbol{p}}$ et l'indicateur de dégradation globale de la structure $\boldsymbol{I}_{\boldsymbol{d}}$ et le mécanisme de ruine et l'état de dégradation des éléments.

\section{Evaluation de la vulnérabilité}

L'évaluation de la vulnérabilité d'une construction nécessite l'estimation des dommages potentiels aux différents types de structures selon les intensités sismiques. Les approches pour l'évaluation de la vulnérabilité sont multiples et peuvent être classées soit selon leur degré de difficulté [3]. L'analyse pushover est l'une des méthodes d'estimation de la vulnérabilité. C'est une analyse statique non linéaire dans laquelle la structure subit des charges latérales suivant un certain modèle prédéfini, en augmentant l'intensité des charges jusqu'à ce que les modes de ruine commencent à apparaitre dans la structure. Elle est basée sur l'hypothèse que la réponse d'une structure a plusieurs degrés de liberté, peut être assimilée à la réponse d'un système à un seul degré de liberté équivalent. Dans ce cas la réponse est contrôlée par un seul mode de vibration et qui reste constante durant la durée du séisme (figure 1).

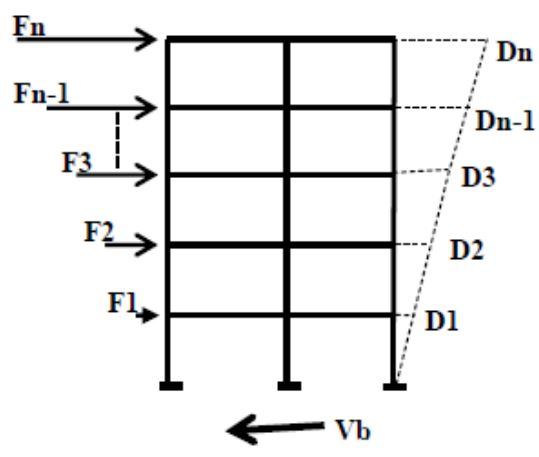

(a) Forces et déplacements dans la structure

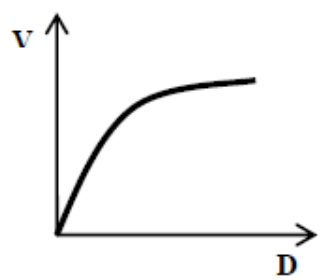

(b) Spectre de capacité, format $\left(\mathrm{V}_{\mathrm{b}}-\mathrm{D}\right)$

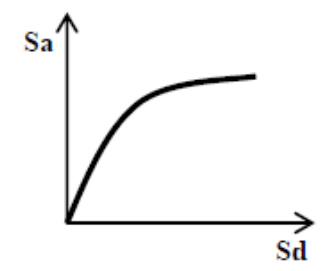

(c) Spectre de capacité, format $\left(\mathrm{S}_{\mathrm{a}}-\mathrm{S}_{\mathrm{d}}\right)$

Fig. 1. Conversion du spectre de capacité.

La force latérale appliquée $\boldsymbol{F}$ et le déplacement $\boldsymbol{D}$ d'un système à un seul de gré de liberté sont donnés par :

$F=\frac{m_{i} \phi_{i}}{\sum_{i=1}^{N} m_{i} \phi_{i}} V_{b}$

$D=\frac{m_{i} \phi_{i}}{\sum_{i=1}^{N} m_{i} \phi_{i}} D_{i}$

Où :

$m_{i}$ : la masse concentrée au niveau $i$.

$\emptyset_{i}$ : Le vecteur déplacement du mode fondamental.

$V_{b}$ : l'effort tranchant à la base.

$D_{i}$ : Le déplacement de la masse $m_{i}$.

La transformation de l'effort tranchant à la base $\boldsymbol{V}_{\boldsymbol{b}}$ en accélération spectrale $\boldsymbol{S}_{\boldsymbol{a}}$, et le déplacement au sommet $\boldsymbol{D}$ en déplacement spectral $\boldsymbol{S}_{\boldsymbol{d}}$, sont donnés par:

$S_{a}=\frac{F}{M_{1}}$

$S_{d}=\frac{D}{\Gamma_{1} \phi_{t, 1}}$

Où :

$M_{1}$ : la masse effective de la construction liée à l'amplitude du premier mode de vibration, et au masses $m_{i}$ des différents niveaux.

$D$ : le déplacement au sommet lié à l'amplitude du premier mode de vibration.

$\Gamma_{l}$ : le facteur de participation modale correspondant au premier mode de vibration.

$\emptyset_{t, 1}$ : amplitude du premier mode de vibration au sommet.

$M_{l}$ et $\Gamma_{l}$ sont données par: 


$$
\begin{gathered}
M_{1}=\frac{\left(\sum_{i=1}^{N} m_{i} \phi_{i, 1}\right)^{2}}{\sum_{i=1}^{N} m_{i} \phi_{i, 1}^{2}} \\
\Gamma_{1}=\frac{\sum_{i=1}^{N} m_{i} \phi_{i, 1}}{\sum_{i=1}^{N} m_{i} \phi_{i, 1}^{2}}
\end{gathered}
$$

Avec ces transformations, nous obtenons la courbe de capacité (figure 2). Le point de croisement entre l'exigence (conversion du spectre $\boldsymbol{S a} \boldsymbol{T} \boldsymbol{T}$ au format $\boldsymbol{S a} \boldsymbol{a} \boldsymbol{S d}$ ) et la résistance (courbe de capacité) fournit le point de performance (fonctionnement) qui décrit l'état d'endommagement de l'ouvrage. Au point de fonctionnement, la demande en énergie à dissiper est gale à la capacité à dissiper de l'énergie et correspond à un état de dommage unique. (a) Courbe de capacité

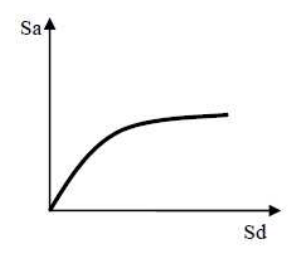

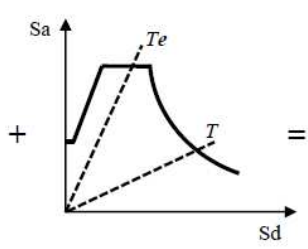

(b) Spectre non linéaire

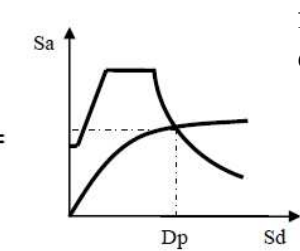

(d) Point de performance
Fig. 2. Point de performance.

La relation force-déplacement utilisée dans SAP 2000 pour définir les zones plastiques est mentionnée sur la figure 3 .

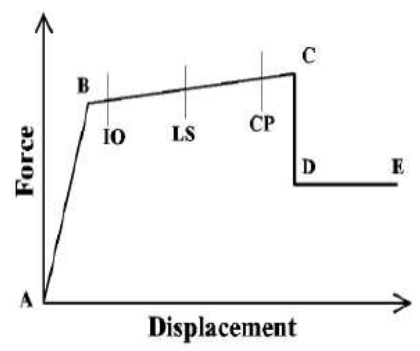

Fig. 3. Relation force-déplacement utilisée dans SAP 2000 pour définir les zones plastiques.

- Le point $\boldsymbol{A}$ correspond aux conditions initiales (non chargement).

- Le point $\boldsymbol{B}$ représente la limite élastique de l'élément.

- L'ordonnée du point $\boldsymbol{C}$ correspond à la résistance nominale et l'abscisse correspondante représente la déformation à partir de laquelle une dégradation marquante de la résistance commence à se produire.

- La baisse manifestant de point $\boldsymbol{C}$ au point $\boldsymbol{D}$ représente la rupture initiale de l'élément.

- La résistance aux charges latérales au-delà du point $\boldsymbol{C}$ est souvent incertaine.

- La résistance résiduelle du point $\boldsymbol{D}$ au point $\boldsymbol{E}$ permet à

l'élément de supporter les charges gravitaires.
- Au-delà du point $\boldsymbol{E}$ qui correspond à la capacité maximale de déformation, les charges gravitaires ne peuvent plus être reprises.

Selon l'ATC 40, le déplacement cible $\boldsymbol{D}_{\text {cible }}$ en fonction de la hauteur de l'ouvrage $\boldsymbol{H}$ est défini par:

$D_{\text {cible }}=\frac{H}{25}$

La demande en ductilité $\boldsymbol{\mu}_{\boldsymbol{D}}$ en fonction du déplacement élastique de la structure $\boldsymbol{D}_{\boldsymbol{y}}$ et le déplacement au point de performance $\boldsymbol{D}_{\boldsymbol{p}}$ est donnée par:

$\mu_{D}=\frac{D_{p}}{D_{y}}$

A mesure que le facteur $\boldsymbol{\mu}_{\boldsymbol{D}}$ est grand, le degré de pénétration de la structure dans le domaine plastique est important.

La raideur initiale (élastique) de la structure $\boldsymbol{K}_{\boldsymbol{e}}$ en fonction de l'effort tranchant élastique $\boldsymbol{V}_{\boldsymbol{y}}$ et le déplacement élastique $\boldsymbol{D}_{\boldsymbol{y}}$ est donnée par:

$$
K_{e}=\frac{V_{y}}{D_{y}}
$$

A mesure que les éléments de la structure se plastifient, la structure subit des dommages qui se traduisent par une diminution de $\boldsymbol{K}_{\boldsymbol{e}}$.

La raideur au point de performance $\boldsymbol{K}_{p}$ en fonction de l'effort tranchant $\boldsymbol{V}_{\boldsymbol{p}}$ et le déplacement $\boldsymbol{D}_{\boldsymbol{p}}$ est donnée par:

$$
K_{p}=\frac{V_{p}}{D_{p}}
$$

L'indicateur de dégradation global $\boldsymbol{I}_{\boldsymbol{d}}$ est donné par :

$$
I_{d}=1-\frac{K_{p}}{K_{e}}
$$

\section{Modélisation des éléments}

Les poutres et les poteaux sont modélisés par des éléments ayant des propriétés élastiques linéaires. Le comportement non linaire des éléments est traduit par l'introduction des rotules plastiques au niveau des sections susceptibles de se plastifier.

Le voile est modélisé par un élément poteau équivalent situé sur l'axe du voile relié à des poutres infiniment rigide. Des rotules plastiques de flexion sont introduites aux extrémités, et une rotule plastique de type (V2 ou V3) au milieu du voile traduit le comportement en cisaillement $[4,5]$. 


\section{Etude d'un bâtiment R+5}

Il s'agit d'une structure en béton armé $\mathrm{R}+5$. Planchers en corps creux de $20 \mathrm{~cm}$ d'épaisseur. Les dimensions sont $(30 \times 35) \mathrm{cm}$ pour les poutres, (30x30) pour les chaînages, (30x40) pour les poteaux et $15 \mathrm{~cm}$ d'épaisseur pour les voiles de contreventement. La hauteur d'étage est de 3,15 m. L'ouvrage est implanté dans une zone sismique de classe II (RPA 99, version 2003), avec un sol de type meuble. Les caractéristiques des matériaux sont les suivantes (Règle BAEL 91):

Béton : $f_{c 28}=25 \mathrm{MPa}, E_{c}=32164 \mathrm{MPa}$.

Aciers : FeE400 pour armature longitudinale, $E_{s}=2.10^{5} \mathrm{MPa}, f_{y}=400 \mathrm{MPa}$.

EeE235 pour armature transversale, $E_{s}=2.10^{5} \mathrm{MPa}, f_{y}=235 \mathrm{MPa}$.

Les charges permanentes $\boldsymbol{G}$ et les surcharges $\boldsymbol{Q}$ sont les suivantes:

$\mathrm{G}_{\text {terrasse }}=0,57 \mathrm{tf} / \mathrm{m}^{2}, Q_{\text {terrasse }}=0,10 \mathrm{tf} / \mathrm{m}^{2}, G_{\text {étage }}=0,50$ $\mathrm{tf} / \mathrm{m}^{2}, Q_{\text {étage }}=0,10 \mathrm{tf} / \mathrm{m}^{2}$

Les différentes variantes de disposition des voiles de contreventement sont mentionnées sur les figures de 4 à 9 pour la variante 1 , de 10 à 15 pour la variante 2 et de 16 à 21 pour la variante 3 .

\section{Variante 1}

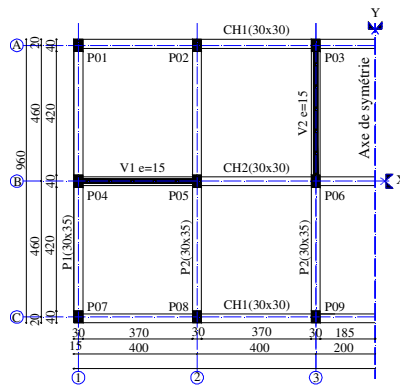

Fig. 4. Variante 1.1

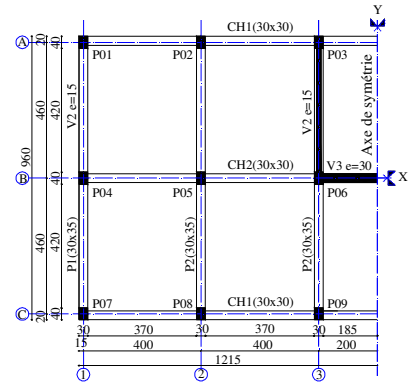

Fig. 6. Variante 1.3

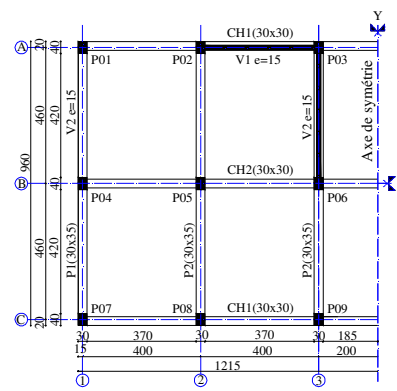

Fig. 8. Variante 1.5

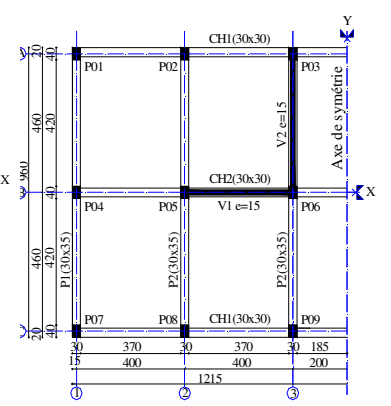

Fig. 5. Variante 1.2

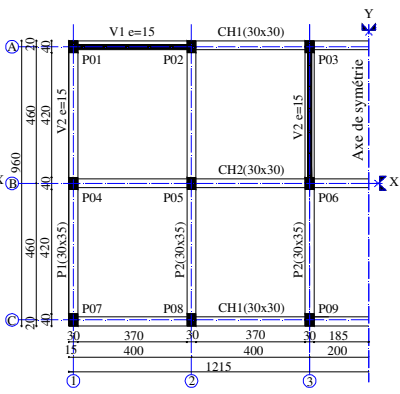

Fig. 7. Variante 1.4

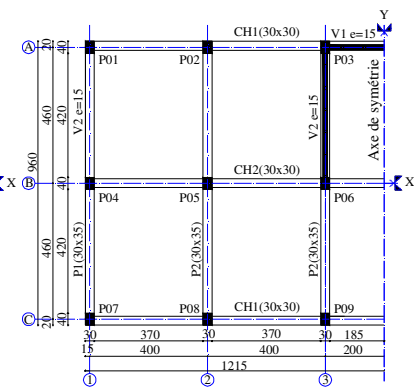

Fig. 9. Variante 1.6

\section{Variante 2}

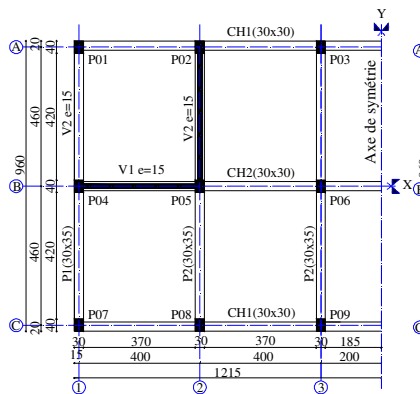

Fig. 10. Variante 2.1

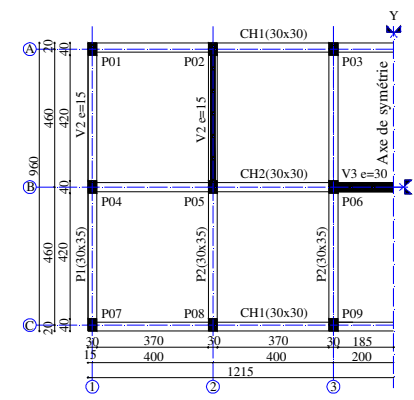

Fig. 12. Variante 2.3

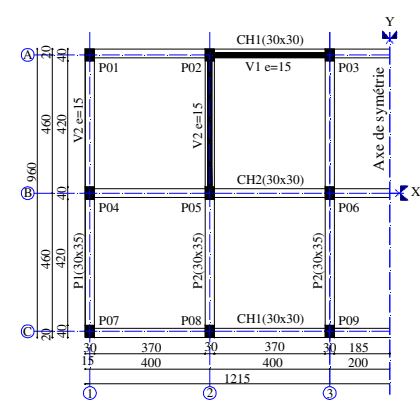

Fig.14. Variante 2.5

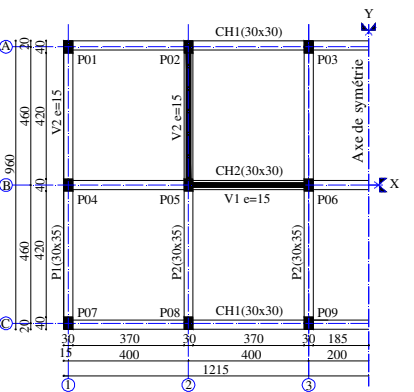

Fig. 11. Variante 2.2

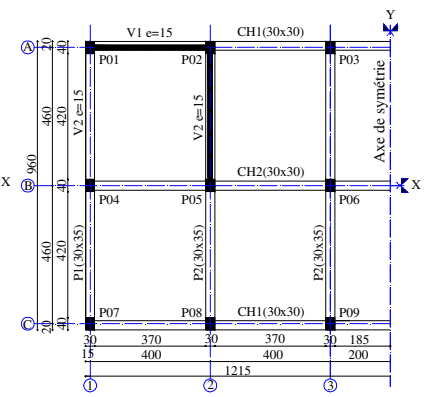

Fig. 13. Variante 2.4

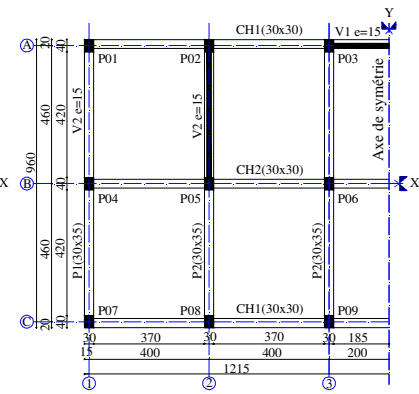

Fig. 15. Variante 2.6

\section{Variante 3}

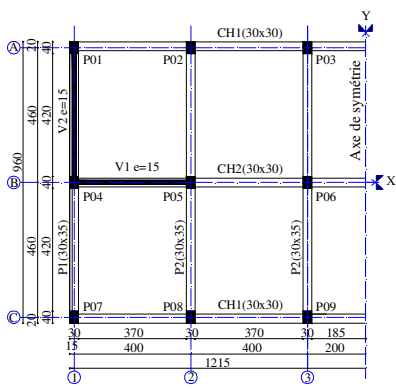

Fig. 16. Variante 3.1

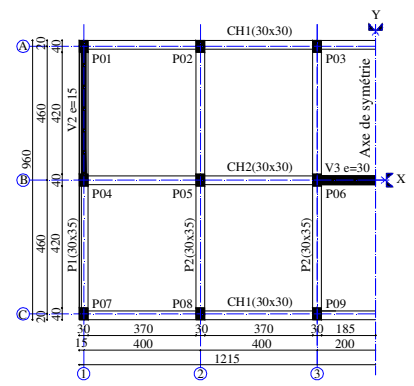

Fig. 18. Variante 3.3

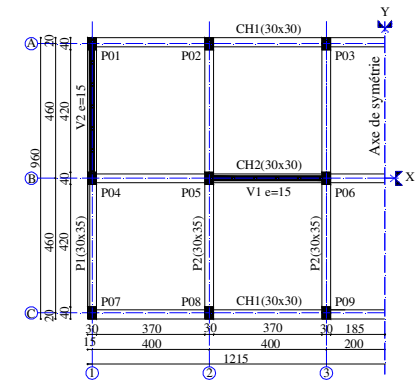

Fig. 17. Variante 3.2

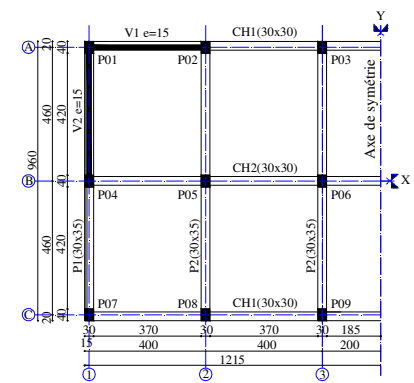

Fig. 19. Variante 3.4 


\section{Analyse des résultats}

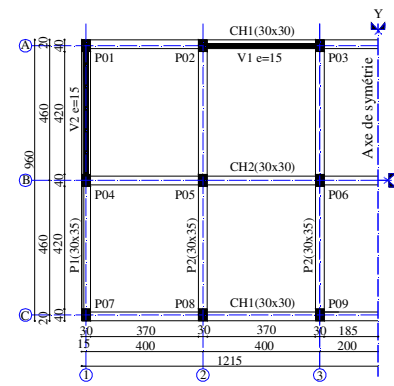

Fig. 20. Variante 3.5

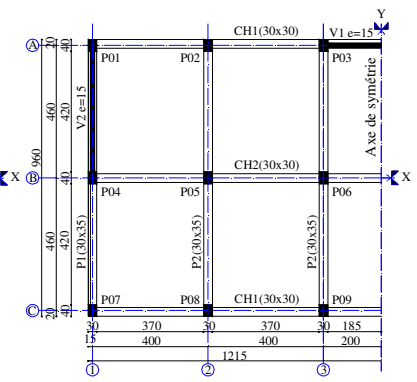

Fig. 21. Variante 3.6

Les résultats de l'analyse dynamique et pushover sont mentionnés sur les tableaux 1et 2 respectivement.

Pour le comportement dynamique de la structure:

- La participation des masses modales est supérieure ou égale à $90 \%$ de la masse totale de la structure,

- Les variantes qui présentent des déplacements purs au premier et deuxième mode de vibrations et une rotation pure au troisième mode sont : V2.6 et V3.1 à V3.6.

Les variantes qui présentent la plus grande participation des masses modales accompagnées de déplacements purs au premier et deuxième mode et une rotation pure au troisième mode sont V3.1 et V3.4, par conséquent la meilleure disposition des voiles de contreventements est celle qui est placées à l'extrémité de la structure avec une symétrie parfaite.

Tableau 1. Résultats de l'analyse dynamique

\begin{tabular}{|c|c|c|c|c|c|c|c|c|c|}
\hline \multirow[t]{2}{*}{ Variante } & \multirow[t]{2}{*}{$\begin{array}{c}\text { Nom } \\
\text { Variante }\end{array}$} & \multirow{2}{*}{$\begin{array}{l}\text { Période } \\
\text { fond. } \\
\text { (s) }\end{array}$} & \multicolumn{2}{|c|}{$\begin{array}{l}\text { Participation } \\
\text { des masses }\end{array}$} & \multicolumn{2}{|c|}{$\begin{array}{c}\text { Délacement } \\
\text { au sommet }(\mathrm{cm})\end{array}$} & \multicolumn{3}{|c|}{$\begin{array}{l}\text { Comportement dynamique } \\
\text { ( } \mathrm{T}: \text { translation, Rot : rotation) }\end{array}$} \\
\hline & & & $\mathrm{X}(\%)$ & $\mathrm{Y}(\%)$ & $\mathrm{X}(\mathrm{cm})$ & $\mathrm{Y}(\mathrm{cm})$ & Mode 1 & Mode 2 & Mode 3 \\
\hline $\begin{array}{c}\text { Structure } \\
\text { sans } \\
\text { voiles } \\
\end{array}$ & $\begin{array}{c}\text { Structure } \\
\text { en } \\
\text { Portique }\end{array}$ & 1,39 & 99 & 99 & 3,04 & 3,40 & $\mathrm{~T}: \mathrm{x}$ & Rot : z & $\mathrm{T}: \mathrm{y}$ \\
\hline \multirow{6}{*}{$\begin{array}{c}\text { Variante } \\
1\end{array}$} & V1.1 & 1,02 & 90 & 90 & 1,13 & 1,20 & Rot: $z$ & $\mathrm{~T}: \mathrm{x}$ et $\mathrm{y}$ & $\mathrm{T}: \mathrm{x}$ et $\mathrm{y}$ \\
\hline & V1.2 & 0,94 & 90 & 91 & 0,94 & 1,76 & Rot : $\mathrm{z}$ & $\mathrm{T}: \mathrm{x}$ et $\mathrm{y}$ & $\mathrm{T}: \mathrm{x}$ et $\mathrm{y}$ \\
\hline & V1.3 & 0,93 & 91 & 91 & 1,03 & 1,03 & Rot : $\mathrm{z}$ & $\mathrm{T}: \mathrm{x}$ et $\mathrm{y}$ & $\mathrm{T}: \mathrm{x}$ et $\mathrm{y}$ \\
\hline & V1.4 & 0,74 & 90 & 90 & 1,15 & 1,18 & Rot: $\mathrm{z}$ & $\mathrm{T}: \mathrm{x}$ et $\mathrm{y}$ & $\mathrm{T}: \mathrm{x}$ et $\mathrm{y}$ \\
\hline & V1.5 & 0,70 & 91 & 91 & 1,01 & 1,18 & Rot : $\mathrm{z}$ & $\mathrm{T}: \mathrm{x}$ et $\mathrm{y}$ & $\mathrm{T}: \mathrm{x}$ et $\mathrm{y}$ \\
\hline & V1.6 & 0,56 & 91 & 91 & 0,91 & 0,93 & $\mathrm{~T}: \mathrm{x}$ et $\mathrm{y}$ & Rot : z & $\mathrm{T}: \mathrm{x}$ et $\mathrm{y}$ \\
\hline & & & & & & & & & \\
\hline \multirow{6}{*}{$\begin{array}{c}\text { Variante } \\
2\end{array}$} & V2.1 & 0,58 & 91 & 91 & 0,93 & 0,95 & $\mathrm{~T}: \mathrm{x}$ et $\mathrm{y}$ & Rot: $\mathrm{z}$ & $\mathrm{T}: \mathrm{x}$ et $\mathrm{y}$ \\
\hline & V2.2 & 0,57 & 91 & 91 & 0,87 & 0,95 & $\mathrm{~T}: \mathrm{x}$ et $\mathrm{y}$ & Rot : z & $\mathrm{T}: \mathrm{x}$ et $\mathrm{y}$ \\
\hline & V2.3 & 0,59 & 89 & 90 & 1,28 & 1,25 & Rot : z & $\mathrm{T}: \mathrm{y}$ & $\mathrm{T}: \mathrm{x}$ \\
\hline & V2.4 & 0,58 & 91 & 91 & 1,06 & 1,21 & $\mathrm{~T}: \mathrm{x}$ et $\mathrm{y}$ & Rot : z & $\mathrm{T}: \mathrm{x}$ et $\mathrm{y}$ \\
\hline & V2.5 & 0,57 & 91 & 91 & 0,94 & 1,02 & $\mathrm{~T}: \mathrm{x}$ et $\mathrm{y}$ & $\mathrm{T}: \mathrm{x}$ et $\mathrm{y}$ & Rot: $\mathrm{z}$ \\
\hline & V2.6 & 0,55 & 90 & 90 & 1,13 & 1,24 & $\mathrm{~T}: \mathrm{X}$ & $\mathrm{T}: \mathrm{y}$ & Rot: $\mathrm{z}$ \\
\hline & & & & & & & & & \\
\hline \multirow{6}{*}{$\begin{array}{c}\text { Variante } \\
\mathbf{3}\end{array}$} & V3.1 & 0,58 & 91 & 91 & 0,93 & 0,99 & $T: x$ & $T: y$ & Rot : $\mathrm{Z}$ \\
\hline & V3.2 & 0,55 & 90 & 90 & 1,07 & 1,24 & $\mathrm{~T}: \mathrm{X}$ & $\mathrm{T}: \mathrm{y}$ & Rot : z \\
\hline & V3.3 & 0,59 & 89 & 90 & 1,28 & 1,24 & $\mathrm{~T}: \mathrm{x}$ & $\mathrm{T}: \mathrm{y}$ & Rot: $\mathrm{z}$ \\
\hline & V3.4 & 0,57 & 91 & 91 & 0,94 & 0,98 & $T: x$ & $T: y$ & Rot : $\mathrm{z}$ \\
\hline & V3.5 & 0,55 & 90 & 90 & 1,13 & 1,26 & $\mathrm{~T}: \mathrm{x}$ & $\mathrm{T}: \mathrm{y}$ & Rot : z \\
\hline & V3.6 & 0,55 & 90 & 90 & 1,13 & 1,24 & $T: x$ & $T: y$ & Rot: $\mathrm{z}$ \\
\hline
\end{tabular}

Tableau 2. Résultats de l'analyse pushover

\begin{tabular}{|c|c|c|c|c|c|c|c|c|c|c|}
\hline \multirow[t]{4}{*}{ Var. } & \multicolumn{10}{|c|}{ Sens y-y } \\
\hline & \multirow{2}{*}{\multicolumn{4}{|c|}{ Courbe de capacité }} & \multirow{2}{*}{\multicolumn{2}{|c|}{$\begin{array}{c}\text { Analyse Pushover } \\
\text { Point de performance }\end{array}$}} & \multirow{2}{*}{\multicolumn{4}{|c|}{ Paramètres }} \\
\hline & & & & & & & & & & \\
\hline & $\begin{array}{l}\mathrm{V}_{\max } \\
\text { (tf) }\end{array}$ & $\begin{array}{l}\mathrm{D}_{\max } \\
(\mathrm{m})\end{array}$ & $\begin{array}{l}\text { Vy } \\
(\mathrm{tf})\end{array}$ & $\begin{array}{l}\text { Dy } \\
(\mathrm{m})\end{array}$ & $\begin{array}{l}\mathrm{Vp} \\
(\mathrm{tf})\end{array}$ & $\begin{array}{l}\text { Dp } \\
(\mathrm{m})\end{array}$ & $\mu_{\mathrm{D}}$ & $\begin{array}{c}\mathrm{Ke} \\
(\mathrm{tf} / \mathrm{m})\end{array}$ & $\begin{array}{c}\mathrm{Kp} \\
(\mathrm{tf} / \mathrm{m})\end{array}$ & $\begin{array}{l}\text { Id } \\
(\%)\end{array}$ \\
\hline \multicolumn{11}{|c|}{ Variante 3} \\
\hline V3.1 & 262,68 & 0,057 & 77,797 & 0,008 & 124,593 & 0,021 & 2,62 & 9724,62 & 5933,00 & 0,39 \\
\hline V3.2 & 261,74 & 0,062 & 74,915 & 0,008 & 123,762 & 0,02 & 2,22 & 9364,37 & 6188,10 & 0,34 \\
\hline V3.3 & 322,17 & 0,067 & 76,068 & 0,008 & 130,722 & 0,02 & 2,50 & 9508,50 & 6536,10 & 0,31 \\
\hline V3.4 & 374,64 & 0,09 & 78,46 & 0,007 & 129,390 & 0,02 & 2,85 & 11208,6 & 6469,50 & 0,42 \\
\hline V3.5 & 384,72 & 0,102 & 76,678 & 0,008 & 127,548 & 0,02 & 2,50 & 9584,75 & 6377,40 & 0,33 \\
\hline V3.6 & 372,27 & 0,093 & 74,712 & 0,008 & 127,833 & 0,02 & 2,50 & 9265 & 6391,65 & 0,31 \\
\hline
\end{tabular}




\section{Conclusion}

La participation des masses modales est supérieure ou égale à $90 \%$ de la masse totale de la structure pour l'ensemble des variantes. Les variantes qui présentent des déplacements purs au premier et deuxième mode de vibrations et une rotation pure au troisième mode sont: V2.6 et V3.1 à V3.6.

Les variantes qui présentent la plus grande participation des masses modales accompagnées de déplacements purs au premier et deuxième mode et une rotation pure au troisième mode sont V3.1 et V3.4.

Les variantes qui présentent un indice de ductilité élevé et une meilleure pénétration dans le domaine plastique sont V3.1 et V3.4. Par conséquent la meilleure disposition des voiles de contreventements est celle qui sont placées à l'extrémité de la structure avec une symétrie parfaite.

\section{Références}

1. Victor Davidovici, Rapport de mission séisme de Boumerdes, Ministère de l'habitat et de l'urbanisme, Algérie, (2003)

2. Milan Zacek, Conception parasismique niveau d'avant projet, Cahier $\mathrm{N}^{\circ} 1$, Collection conception parasismique, (2004)

3. Arie-José Nollet, Evaluation de la vulnérabilité des batiments existants, Département de genie de la construction, (2004)

4. K. Galal, H. El-Sokkary, Advanced in modelling of $R C$ shear walls, The 14 th World conference on earthquake engineering, Octoer 12-17, Bejin, China, (2008)

5. Matej Fischinger, Tajana Isacovic, Benchmark analysis of structural wall, The $12^{\text {th }}$ world conference on earthquake engineering, Auckland, Nouvelle Zelande, (2000) 